\title{
Cycle ergometer and inspiratory muscle training offer modest benefit compared with cycle ergometer alone: a comprehensive assessment in stable COPD patients
}

\author{
This article was published in the following Dove Press journal: \\ International Journal of COPD \\ 6 September 2017 \\ Number of times this article has been viewed
}

\section{Kai Wang, I,* Guang-qiao Zeng, 2,* Rui Li, ',*Yu-wen Luo,' Mei Wang,' Yu-he $\mathrm{Hu}$,' Wen-hui Xu,' Lu-qian Zhou, ${ }^{2}$ Rong-chang Chen, ${ }^{2}$ Xin Chen' \\ 'Department of Respiratory Medicine, Zhujiang Hospital, Southern Medical University, Guangzhou, China; ${ }^{2}$ Department of Respiratory Medicine, The State Key Laboratory of Respiratory Disease, National Clinical Research Center for Respiratory Disease, Guangzhou Institute of Respiratory Disease, First Affiliated Hospital of Guangzhou Medical University, Guangzhou, China \\ *These authors contributed equally to this work}

Correspondence: Xin Chen Department of Respiratory Medicine, Zhujiang Hosptial, Southern Medical University, 253 Gongye Road, Guangzhou 510282, China

Tel +86 2062782296

Fax +86 2061643010

Email chen_xin1020@163.com

Rong-chang Chen

Department of Respiratory Medicine,

The State Key Laboratory of Respiratory Disease, National Clinical Research

Center for Respiratory Disease, Guangzhou Institute of Respiratory Disease, First Affiliated Hospital of Guangzhou Medical University, I5 I Yanjiang Road, Guangzhou 510120, China Email chenrc@vip.I63.com
Background: Cycle ergometer training (CET) has been shown to improve exercise performance of the quadriceps muscles in patients with COPD, and inspiratory muscle training (IMT) may improve the pressure-generating capacity of the inspiratory muscles. However, the effects of combined CET and IMT remain unclear and there is a lack of comprehensive assessment.

Materials and methods: Eighty-one patients with COPD were randomly allocated to three groups: 28 received 8 weeks of CET + IMT (combined training group), 27 received 8 weeks of CET alone (CET group), and 26 only received 8 weeks of free walking (control group). Comprehensive assessment including respiratory muscle strength, exercise capacity, pulmonary function, dyspnea, quality of life, emotional status, nutritional status, and body mass index, airflow obstruction, and exercise capacity index were measured before and after the pulmonary rehabilitation program.

Results: Respiratory muscle strength, exercise capacity, inspiratory capacity, dyspnea, quality of life, depression and anxiety, and nutritional status were all improved in the combined training and CET groups when compared with that in the control group $(P<0.05)$ after pulmonary rehabilitation program. Inspiratory muscle strength increased significantly in the combined training group when compared with that in the CET group $\left(\triangle \mathrm{PI} \mathrm{max}_{\max }[\right.$ maximal inspiratory pressure] $5.20 \pm 0.89 \mathrm{cmH}_{2} \mathrm{O}$ vs $\left.1.32 \pm 0.91 \mathrm{cmH}_{2} \mathrm{O} ; P<0.05\right)$. However, there were no significant differences in the other indices between the two groups $(P>0.05)$. Patients with weakened respiratory muscles in the combined training group derived no greater benefit than those without respiratory muscle weakness $(P>0.05)$. There were no significant differences in these indices between the patients with malnutrition and normal nutrition after pulmonary rehabilitation program $(P>0.05)$.

Conclusion: Combined training is more effective than CET alone for increasing inspiratory muscle strength. IMT may not be useful when combined with CET in patients with weakened inspiratory muscles. Nutritional status had slight impact on the effects of pulmonary rehabilitation. A comprehensive assessment approach can be more objective to evaluate the effects of combined CET and IMT.

Keywords: chronic obstructive pulmonary disease, pulmonary rehabilitation, cycle ergometer, inspiratory muscle training, comprehensive assessment, exercise performance

\section{Plain language summary}

Why was the study done? Cycle ergometer training (CET) and inspiratory muscle training (IMT) - the two pulmonary rehabilitation methods have been shown to benefit patients with COPD. But the additional benefit of IMT when given as an adjunct to CET remains unclear. 
What did the researchers do and find? We enrolled and divided 81 COPD patients into three groups: the combined training group received 8 weeks CET + IMT; the CET training group received 8 weeks CET; the control group received 8 weeks free walking. Then we took a comprehensive approach in evaluating the effects of these training sessions. We found that CET + IMT was more effective than CET alone in increasing inspiratory strength, but not the other aspects. Patients with weakened respiratory muscles in the combined training group derived similar effects to those without respiratory muscle weakness after training. Patients with malnutrition and normal nutrition shared the same effects after rehabilitation.

What do these results mean? CET + IMT offers modest benefit compared with cycle ergometer alone except in increasing inspiratory muscle strength. Combined training may not bring additional effects in patients with weakened inspiratory muscles. Nutritional status had slight impact on pulmonary rehabilitation.

\section{Introduction}

COPD is a common and complicated airway disorder which has become the fourth leading cause of death worldwide. The multi-system involvement of COPD may result in impaired exercise capacity, poor health-related quality of life, and mental problems, including depression and anxiety. ${ }^{1-4}$ For COPD patients, pulmonary rehabilitation has been shown to improve symptoms, exercise tolerance, and well-being. Among diverse approaches to pulmonary rehabilitation, cycle ergometer training (CET) may optimally enhance the quadriceps muscle functions, significantly improve exercise performance, dyspnea, and quality of life, and therefore has been accepted as an important part of pulmonary rehabilitation. ${ }^{4}$ Apart from CET, studies also confirmed that inspiratory muscle training (IMT) may increase inspiratory muscle strength and reduce the severity of dyspnea in COPD patients. ${ }^{5,6}$ Despite the beneficial effects of IMT on inspiratory muscle strength and endurance, its additional benefits on exercise performance and quality of life did not seem to be significant. ${ }^{7}$ As such, future studies in COPD patients should focus specifically on the effect of IMT or IMT + other rehabilitative interventions compared to other IMT-excluded rehabilitations for outcome assessments. Unfortunately, due to the limited sample sizes and mixed conclusions of previous studies, the benefit of IMT in pulmonary rehabilitation when given as an adjunct to CET remains unclear. ${ }^{7-11}$ Furthermore, in these published studies, a comprehensive outcome assessment approach for the combined training has rarely been performed, which should ideally account for more parameters such as fat-free mass index (FFMI), body mass index (BMI), airflow obstruction, dyspnea, and exercise capacity index (BODE), depression and anxiety, COPD Assessment Test (CAT), modified Medical Research Council (mMRC), and
St George's Respiratory Questionnaire (SGRQ) rather than merely focus on the resultant benefits for muscle function and exercise endurance. In light of the reference to pulmonary rehabilitation as a comprehensive intervention by the American Thoracic Society/European Respiratory Society (ATS/ERS 2013) statement, ${ }^{4}$ we speculated that a comprehensive approach would be much more worthwhile when evaluating the effects of rehabilitation in COPD patients.

Given that both CET and IMT may be therapeutically beneficial for post-clinical COPD patients in whom damage to the quadriceps muscles and inspiratory muscles is associated with poor exercise performance and life quality, ${ }^{12}$ we hypothesized that a combination of the two may improve the outcome of pulmonary rehabilitation. The aim of this study was to comprehensively evaluate whether CET + IMT would lead to additional benefits compared with CET alone in COPD patients. In addition, the effect of pulmonary rehabilitation was compared between patients with and without respiratory muscle weakness.

\section{Materials and methods Study design}

This was a prospective randomized controlled study conducted in a rehabilitation center at Zhujiang Hospital affiliated to Southern Medical University (Guangzhou, China) between April 1, 2015 and October 1, 2015. Reporting of this study was in accordance with the Consolidated Standard of Reporting Trials (CONSORT) statement. ${ }^{13}$ The study protocol rigorously followed the Declaration of Helsinki and Committee on Publication Ethics (COPE) guidelines, and was approved by the Ethics Committee of Zhujiang Hospital, Southern Medical University. All participants in this study signed a written informed consent. Prior to the start of this trial, the study protocol was registered online (register ClinicalTrials.gov, number: NCT02285400).

\section{Recruitment of patients}

The inclusion criteria were: age $>40$ years; diagnosis of stable COPD based on the Global Initiative for Chronic Obstructive Lung Disease (GOLD) guidelines; ${ }^{1}$ and no participation in any pulmonary rehabilitation program in the previous 2 months. To be included in this study, the participants had to be able to understand the investigator's instructions and complete the tests required in the study. Patients were excluded if they had acute or chronic airway diseases other than COPD, cardiovascular disorders (such as acute coronary syndrome), metabolic conditions (such as diabetes or hyperthyroidism), or other health problems that would interfere with exercise performance or the testing procedures. 
Potentially eligible patients were recruited from the outpatient clinic of Southern Medical University Zhujiang Hospital. They were informed about the aim and procedure of this study. Within a half-year run-in period, we approached 261 consecutive patients with COPD, of whom 121 met our inclusion criteria and all were instructed to do a 30-minute free walk on the exercise area of our rehabilitation center three times weekly. During this period, the patients were allowed to quit and exit the study. Regular medication for COPD based on GOLD guidelines was also allowed during this period and throughout the study. At the end of the run-in period, 81 eligible patients remaining on free walk (16 females and 65 males; mean age $70.32 \pm 5.878$ years) were enrolled in the trial. These 81 patients were randomized into three groups (CET + IMT group, CET group, and control group) using computer-generated random numbers at a 1:1:1 allocation ratio. The randomization and allocation was completed independently by a research nurse according to a random number sealed in opaque envelopes.

\section{Study interventions}

The CET group received 8 weeks of CET alone, and the combined training group received 8 weeks of CET + IMT . The control group received 8 weeks of free walking as in the run-in period. All training was performed under the supervision of an experienced respiratory therapist. Throughout the study period, these subjects were on a standardized diet as prepared and guided by a designated dietitian according to the previous nutritional statement. ${ }^{14}$

Patients in the CET group received 30-minute CET three times per week for 8 weeks. The CET was performed on an electromechanically braked cycle (model E100 P/K, Cosmed, Rome, Italy) in our rehabilitation center. The exercise intensity threshold in the lower limbs was calculated as $70 \%$ maximal oxygen consumption $\left(\mathrm{VO}_{2 \max }\right)$ during cardiopulmonary exercise testing (CPET). Interval training protocol was used whereby patients performed three workout sets, each 10 minutes in duration and separated by 3 - to 5 -minute rest intervals of unloaded cycling. ${ }^{15}$ The training workload was evaluated weekly. We selected loads that patients described as "somewhat hard", ie, graded 12-14 on the Rating of Perceived Exertion (RPE) scale. ${ }^{16}$

Patients in the combined training group received 30-minute CET and 14-minute IMT three times per week for 8 weeks. The training sessions were separated such that the patients performed CET first as described previously, and then IMT after a 30-minute rest period from the end of CET. The IMT was given with a threshold-loaded IMT device (model HS730, Philips, Amsterdam, the Netherlands). Training was initiated at an intensity that was $30 \%$ of maximal inspiratory pressure ( $\mathrm{PI}_{\max }$ ) and had been tested with a digital manometer. Interval training protocol was used whereby patients performed seven workout sets, each 2 minutes in duration, separated by rest intervals lasting 1 minute. ${ }^{5}$ The training load was adjusted weekly: the load was increased while maintaining an RPE grade of 12-14.

Patients in the control group completed a 30-minute free walk three times per week on the playground of the rehabilitation center, for 8 more weeks. As in the run-in period, there was no requirement for speed and distance. The patients were free to stop for a while if they felt uncomfortable due to dyspnea or fatigue, and go on walking when the symptoms subsided.

The severity of dyspnea was evaluated during exercise using the Borg scale. ${ }^{17}$ Before the exercises, the Borg scale was explained to patients. To ensure patient safety and to guide the adjustment of training load, we monitored the following indicators during the training sessions to make sure that they were within permissible ranges: heart rate $(\mathrm{HR})<$ maximum HR obtained by CPET or $90 \%$ of predicted maximum (calculated as $90 \% \times[220$ - age in years $]$ ); pulse oxygen saturation $\left(\mathrm{SPO}_{2}\right) \geq 85 \%$; blood pressure $(\mathrm{BP}) \leq 200 / 100 \mathrm{mmHg}$; Borg scale $\leq 5$; RPE grade $<15$ (tired). When any of these indicators exceeded the permissible range, the training session was suspended immediately, and the patient was instructed to have a rest and further evaluated by a physician for the need to terminate the training of the day or receive medications before proceeding with training.

\section{Outcome measures}

A comprehensive assessment of pulmonary rehabilitation was performed based on respiratory muscle strength, exercise capacity, pulmonary function, symptoms, nutritional status, mental status, and quality of life, using a number of measures closely relevant to COPD as described in the following paragraphs. The primary outcome measures were respiratory muscle strength and exercise capacity. The secondary outcome measures were pulmonary function, symptoms, nutritional status, mental status, and quality of life. All tests were conducted by an experienced respiratory therapist who was blinded to the study protocol. All these data were measured or recorded prior to the start (baseline) and within 2 days after the end of the study intervention.

\section{Respiratory muscle strength}

Respiratory muscle strength was determined according to $\mathrm{PI}_{\max }$ and maximal expiratory pressure $\left(\mathrm{PE}_{\max }\right)$ using a digital manometer (AZ-8205; AZ Instruments, Taichung 
City, Taiwan). The testing procedures were performed in adherence with the ATS/ERS standardization protocols. ${ }^{18}$ Both $\mathrm{PI}_{\max }$ and $\mathrm{PE}_{\max }$ were measured at least three times, and the highest result was used for analysis. The testing error was required to be less than $20 \%$ and the interval between two consequent measurements was no less than 30 seconds. The lower limit of normal value for $\mathrm{PI}_{\max }$ was $60 \mathrm{cmH}_{2} \mathrm{O}$.

\section{Exercise capacity}

Exercise capacity was evaluated using the 6-minute walk test (6MWT) and a maximal exercise test. The 6MWT was performed for $30 \mathrm{~m}$ on a flat surface. ${ }^{19}$ Oxyhemoglobin saturation and HR were monitored by pulse oximetry. The largest values measured over 2 days were chosen for analysis. Oxygen saturation was maintained at more than $90 \%$ during testing in all subjects. The patients used the Borg scale to rate dyspnea at the end of the 6MWT.

The maximal exercise test was evaluated the next day after 6MWT. The maximal exercise test was measured during standard $5 \mathrm{~W} /$ minute or $10 \mathrm{~W} /$ minute incremental symptom-limited cycle ergometer testing protocol using a CPET machine (Quark-PFT Ergo, Cosmed). ${ }^{20}$ The protocol included three stages comprising: a rest stage (5 minutes of warming-up with a zero workload); an exercise stage (according to the exercise capacity of each patient to ensure the rate of workload progression, using a ramp protocol to increase the workload, keeping at 55-65 rpm until maximal exertion); and a recovery stage (3-5 minutes of recovery at zero workload). The measured CPET parameters included oxygen uptake $\left(\mathrm{VO}_{2}\right)$, carbon dioxide output $\left(\mathrm{VCO}_{2}\right)$, respiratory exchange ratio (RER), $\mathrm{SPO}_{2}$, and $\mathrm{HR}$, as well as ventilatory pattern parameters including minute ventilation (VE), respiratory frequency (Rf), and tidal volume (VT). These parameters were measured at the following time points: at rest before beginning exercise; at the end of warm-up stage; at the anaerobic threshold (AT); at the peak. The patients used the Borg scale to rate dyspnea every minute. Progressive incremental exercise testing was ended when the following situation was shown in the patients: chest pain suggestive of ischemia; $\mathrm{SPO}_{2} \leq 80 \%$ accompanied by symptoms and signs of severe hypoxemia; notable ischemic electrocardiogram changes and arrhythmia; fall in systolic pressure $>20 \mathrm{mmHg}$ from the highest value during the test; or hypertension (>250 mmHg systolic; $>120 \mathrm{mmHg}$ diastolic) ${ }^{20}$

\section{Pulmonary function}

Pulmonary function was measured using a spirometer (Pony FX229, Cosmed), which was calibrated daily, and in accordance with the ATS guidelines. ${ }^{21}$ All baseline functional measures (including forced expiratory volume in 1 second $\left[\mathrm{FEV}_{1}\right], \mathrm{FEV}_{1}$ percent predicted [\% $\%$ red], forced vital capacity [FVC], FVC \% pred, FEV ${ }_{1} /$ FVC, maximal voluntary ventilation [MVV], and inspiratory capacity [IC]) were recorded before and after a bronchodilator test. All lung function parameters were compared with the reference values published by the European Community for Steel and Coal. ${ }^{22}$

\section{Dyspnea, quality of life, and depression and anxiety}

The severity of dyspnea was evaluated using the mMRC and the CAT. ${ }^{23,24}$ Quality of life was evaluated using the SGRQ. ${ }^{25}$ Depression and anxiety were evaluated using the Hospital Anxiety and Depression Scale (HADS), which includes 14 questions (seven for depression and seven for anxiety). ${ }^{26}$ All questionnaires were administered by an experienced investigator.

\section{Nutritional status}

Nutritional status was measured using the BMI (calculated as body mass/height ${ }^{2}$ ) and FFMI (calculated as fat-free mass/ height $^{2}$ ). FFMI was measured twice by multifrequency and 8 -spot electrodes, and the average value was chosen for analysis. We defined a low FFMI as being $\leq 16 \mathrm{~kg} / \mathrm{m}^{2}$ in men and $\leq 15 \mathrm{~kg} / \mathrm{m}^{2}$ in women. ${ }^{27,28}$

\section{Statistical analysis}

An intention-to-treat protocol was used for data analysis. For the primary analyses, we used the last observation carried forward (LOCF) imputation method for data of the drop-outs. The statistical analyses were performed using Statistical Package for the Social Sciences version 19.0 software (IBM Corporation, Armonk, NY, USA). Patient characteristics at baseline, shown in Table 1, were presented as mean $\pm \mathrm{SD}$. The values in other tables and figures were described as mean \pm SE. The baseline differences between groups were compared using one-way analysis of variance (ANOVA). Based on general linear models, we used analysis of covariance models (ANCOVA) to analyze the difference of primary and secondary outcomes (after minus before intervention, shown as $\Delta$ ), adjusting the models with least-significant difference (LSD) correction for the baseline score. We used a repeated measures ANOVA to compare the changes of ventilatory pattern parameters and Borg scores measured during CPET between groups as shown in Figure 1. Subgroup analyses were also performed using ANCOVA with LSD adjustment. A $P$-value $<0.05$ was considered statistically significant. We performed a sensitivity analysis with the same result. 
Table I Patient characteristics at baseline

\begin{tabular}{|c|c|c|c|c|c|}
\hline Parameter & $\begin{array}{l}\text { Control group } \\
(n=26)\end{array}$ & $\begin{array}{l}\text { CET group } \\
(n=27)\end{array}$ & $\begin{array}{l}\text { Combined } \\
\text { training group } \\
(n=28)\end{array}$ & $F$ & $P$-value \\
\hline Age (years) & $69.8 \pm 6.4$ & $70.0 \pm 6.3$ & $70.8 \pm 4.5$ & 0.237 & 0.790 \\
\hline $\mathrm{PI}_{\max }\left(\mathrm{cmH}_{2} \mathrm{O}\right)$ & $63.81 \pm 23.73$ & $74.66 \pm 13.83$ & $72.40 \pm 20.41$ & 2.227 & 0.115 \\
\hline $\mathrm{PE}_{\max }\left(\mathrm{cmH}_{2} \mathrm{O}\right)$ & $64.74 \pm 39.09$ & $77.24 \pm 30.03$ & $77.40 \pm 35.82$ & 1.132 & 0.327 \\
\hline 6MWD (m) & $525 \pm 70$ & $511 \pm 77$ & $538 \pm 60$ & 1.101 & 0.338 \\
\hline $\mathrm{VO}_{2 \max }(\mathrm{mL} / \mathrm{min})$ & $I, 01 I \pm 390$ & $\mathrm{I}, \mathrm{I} 54 \pm 297$ & $994 \pm 272$ & 2.015 & 0.140 \\
\hline $\mathrm{VO}_{2 \max } \%$ pred (\%) & $61.83 \pm 22.23$ & $66.81 \pm 16.64$ & $65.25 \pm 15.61$ & 0.492 & 0.613 \\
\hline $\mathrm{VO}_{2 \max } / \mathrm{kg}(\mathrm{mL} / \mathrm{min} / \mathrm{kg})$ & $17.01 \pm 6.24$ & $18.13 \pm 4.82$ & $17.18 \pm 3.94$ & 0.374 & 0.689 \\
\hline AT (mL/min) & $823 \pm 321$ & $923 \pm 197$ & $804 \pm 188$ & 1.912 & 0.155 \\
\hline AT\%pred (\%) & $50.75 \pm 19.67$ & $53.59 \pm 11.62$ & $52.93 \pm 11.26$ & 0.266 & 0.767 \\
\hline FVC (L) & $2.69 \pm 0.70$ & $2.78 \pm 0.66$ & $2.4 I \pm 0.62$ & 2.276 & 0.110 \\
\hline FVC\%pred (\%) & $84.15 \pm 20.56$ & $80.89 \pm 18.73$ & $75.21 \pm 16.19$ & 1.620 & 0.204 \\
\hline $\mathrm{FEV}_{1}(\mathrm{~L})$ & $1.42 \pm 0.46$ & $1.33 \pm 0.47$ & $1.19 \pm 0.40$ & 1.959 & 0.148 \\
\hline FEV,\%pred (\%) & $57.54 \pm 18.98$ & $51.26 \pm 18.00$ & $49.82 \pm 16.14$ & 1.429 & 0.246 \\
\hline $\mathrm{FEV}_{1} / \mathrm{FVC}$ & $53.19 \pm 10.18$ & $47.23 \pm 9.90$ & $49.60 \pm 11.18$ & 2.180 & 0.120 \\
\hline MVV (L) & $51.33 \pm 17.92$ & $52.65 \pm 18.21$ & $46.54 \pm 18.75$ & 0.850 & 0.431 \\
\hline IC (L) & $1.77 \pm 0.52$ & $1.90 \pm 0.46$ & $|.7| \pm 0.53$ & 0.994 & 0.375 \\
\hline $\mathrm{mMRC}$ & $1.19 \pm 0.85$ & $I .4 I \pm 0.84$ & $\mathrm{I} .29 \pm 0.8 \mathrm{I}$ & 0.444 & 0.643 \\
\hline CAT & $7.96 \pm 5.24$ & $8.93 \pm 5.21$ & $8.93 \pm 5.64$ & 0.285 & 0.753 \\
\hline SGRQ & $12.62 \pm 7.40$ & $14.93 \pm 8.82$ & $16.14 \pm 6.79$ & 1.446 & 0.242 \\
\hline HADS & $5.27 \pm 3.26$ & $4.85 \pm 2.70$ & $5.50 \pm 4.35$ & 0.239 & 0.788 \\
\hline HADS depression & $3.00 \pm 2.28$ & $2.44 \pm 1.72$ & $3.54 \pm 3.23$ & 1.309 & 0.276 \\
\hline HADS anxiety & $2.27 \pm 2.05$ & $2.4 I \pm I .28$ & $1.96 \pm 1.64$ & 0.501 & 0.608 \\
\hline BMI $\left(\mathrm{kg} / \mathrm{m}^{2}\right)$ & $21.31 \pm 3.78$ & $22.40 \pm 2.85$ & $21.31 \pm 2.76$ & 1.071 & 0.348 \\
\hline FFMI $\left(\mathrm{kg} / \mathrm{m}^{2}\right)$ & $16.65 \pm 1.92$ & $16.86 \pm 1.54$ & $16.24 \pm 1.36$ & $\mathrm{I} .075$ & 0.346 \\
\hline BODE & $1.81 \pm 1.36$ & $2.33 \pm 1.90$ & $2.46 \pm 1.58$ & 1.211 & 0.303 \\
\hline
\end{tabular}

Notes: Data are presented as mean \pm SD unless otherwise stated; $P$-value results of analysis of variance (ANOVA) comparison between three groups at baseline.

Abbreviations: CET, cycle ergometer training; $\mathrm{PI}_{\max }$, maximal inspiratory pressure; $\mathrm{PE}_{\max }$, maximal expiratory pressure; 6MWD, 6-minute walking distance; $V \mathrm{O}_{2 \max }$, maximal oxygen consumption; AT, anaerobic threshold; FVC, forced vital capacity; FEV , forced expiratory volume in I second; \%pred, percent predicted; MVV, maximal voluntary ventilation; IC, inspiratory capacity; mMRC, modified Medical Research Council; CAT, COPD Assessment Test; SGRQ, St George's Respiratory Questionnaire; HADS, Hospital Anxiety and Depression Scale; BMI, body mass index; FFMI, fat-free mass index; BODE, body mass index, airflow obstruction, dyspnea, and exercise capacity index.

\section{Results}

Of the 81 patients initially included in the study, not all completed the whole training program. The number of dropouts was similar in the three training groups. Dropouts were due to: lost to follow-up (one from each group), refusal to continue the intervention (one from each group), and worsening of COPD (one each in the CET group and combined training group). Despite these dropouts, with an intention-to-treat analysis and the LOCF imputation method, our analysis accounted for all the 81 participants in this study coming from the control group $(n=26)$, CET group $(n=27)$, and the combined training group ( $\mathrm{n}=28$ ) (Figure 2). The baseline characteristics of the patients were comparable among the three groups, as summarized in Table 1. For patients who remained throughout the study, all training sessions were completed as scheduled, and none of the daily training sessions was terminated due to patient discomfort, dyspnea, or abnormality of physiological parameters (monitored for patient safety during the exercise).

\section{Effect of pulmonary rehabilitation on respiratory muscle strength}

The CET group and the combined training group demonstrated a significant increase in $\mathrm{PI}_{\max }$ and $\mathrm{PE}_{\max }$ when compared with the control group $(P<0.05)$. As shown in Table 2, the differences in $\triangle \mathrm{PI}_{\text {max }}$ between the control group, CET group, and combined training group $\left(-2.38 \pm 0.94 \mathrm{cmH}_{2} \mathrm{O}\right.$, $1.32 \pm 0.91 \mathrm{cmH}_{2} \mathrm{O}$, and $5.20 \pm 0.89 \mathrm{cmH}_{2} \mathrm{O}$, respectively) were statistically significant $(P<0.05)$. Table 2 also shows a significant increase in inspiratory muscle strength in the combined training group when compared with the other two groups. However, there was no significant differences in $\triangle \mathrm{PE}_{\max }$ between the CET group and the combined training group $(P>0.05)$. 

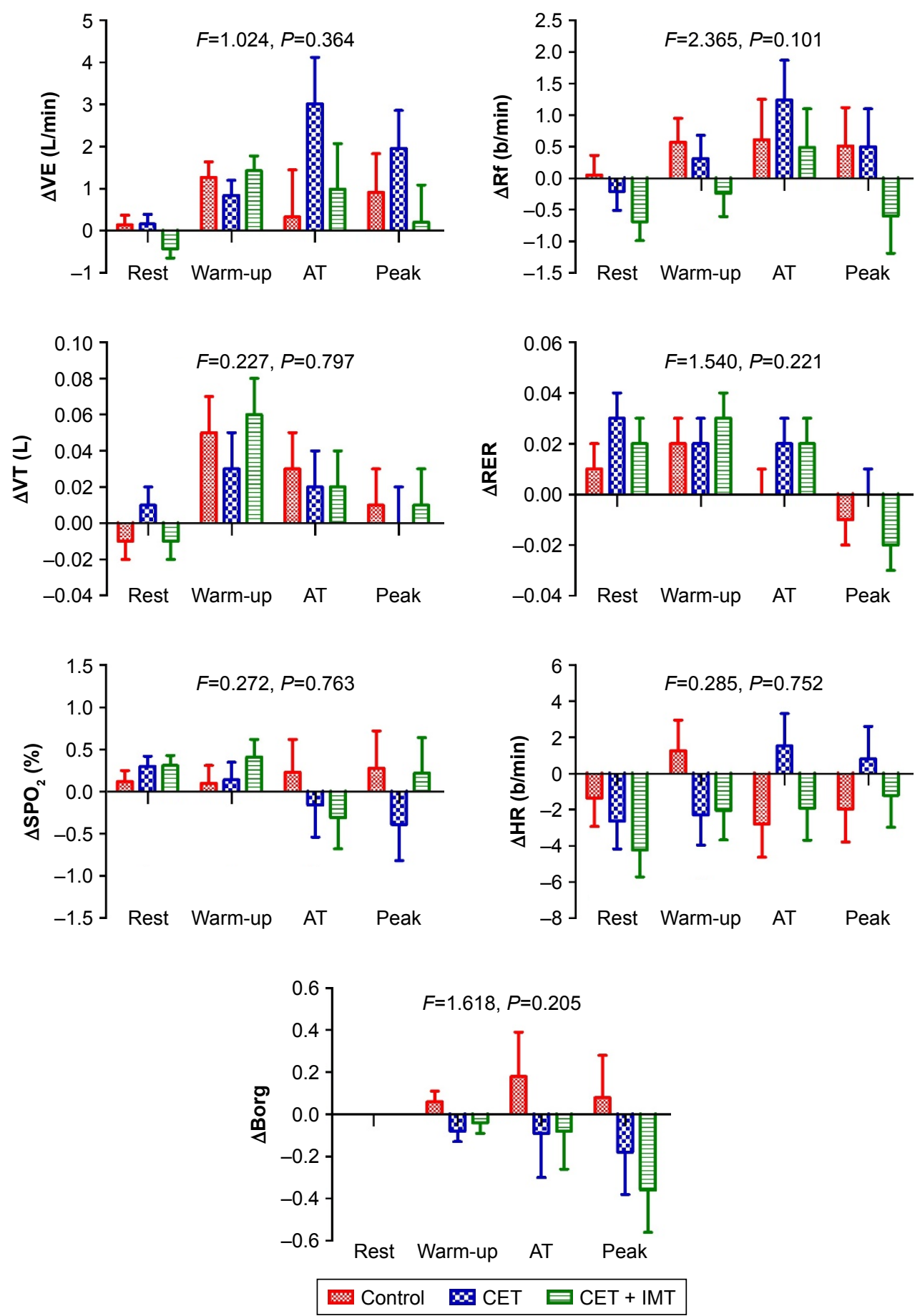

Figure I Changes of ventilatory pattern, $\mathrm{HR}, \mathrm{SPO}_{2}$, and Borg scale during CPET.

Notes: Data are presented as mean \pm SE unless otherwise stated; $\Delta$, difference (after minus before intervention); $P$-value results of analysis of repeated measures analysis of variance (ANOVA) comparison of the differences between groups.

Abbreviations: CET, cycle ergometer training; IMT, inspiratory muscle training; AT, anaerobic threshold; VE, minute ventilation; Rf, respiratory frequency; VT, tidal volume; $\mathrm{SPO}_{2}$, pulse oxygen saturation; $\mathrm{HR}$, heart rate; RER, respiratory exchange ratio; CPET, cardiopulmonary exercise testing.

\section{Effects of pulmonary rehabilitation on exercise capacity}

At baseline, there was no significant difference in exercise capacity between groups. After training, no significant changes of the exercise capacity indices were found in control group. There were significant increases from baseline in the 6MWT, $\mathrm{VO}_{2 \max }, \mathrm{VO}_{2 \max }$ \%pred, $\mathrm{VO}_{2 \max } / \mathrm{kg}, \mathrm{AT}, \mathrm{AT}$ \%pred and workload (all $P<0.05$ ) in both CET group and combined training group (Table 3 ). However, the increases in these indicators did not differ between CET group and 


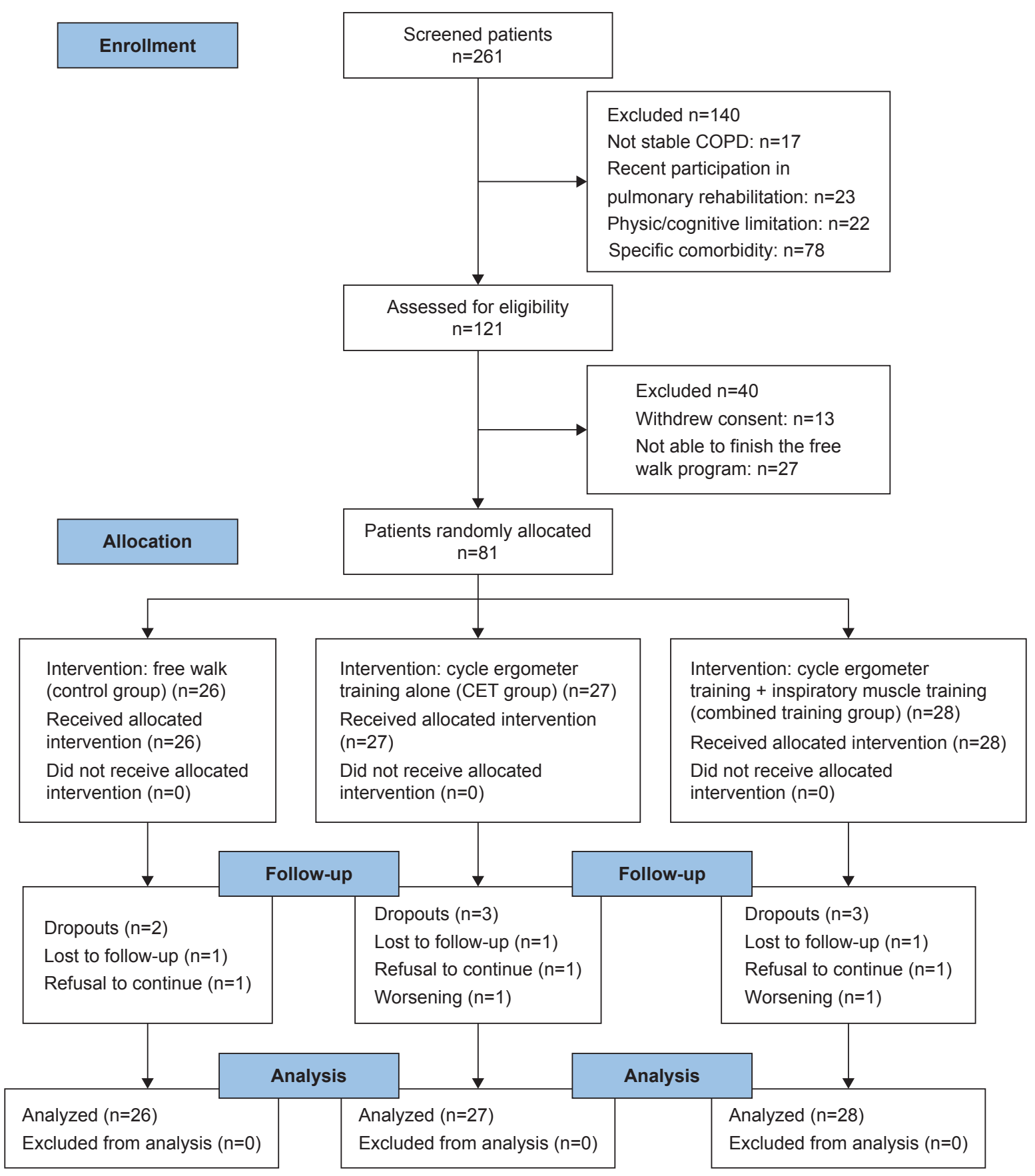

Figure 2 Flow diagram for studies included.

Abbreviation: CET, cycle ergometer training.

combined training group $(P>0.05)$. At the end of 6-minute walking distance test and the measured time points of CPET, the Borg score demonstrated no significant changes in all groups after training. At baseline, there were no differences in VE, VT, Rf, RER, HR, and $\mathrm{SPO}_{2}$ between groups. These parameters demonstrated no significant improvement after training in all groups (Table 3). During CPET, there were no significant differences in these parameters at all time points, as shown in Figure 1.

\section{Effects of pulmonary rehabilitation on static pulmonary function}

There were no changes in FVC, FVCpred, $\mathrm{FEV}_{1}, \mathrm{FEV}_{1}$ pred, $\mathrm{FEV}_{1} / \mathrm{FVC}$, and MVV (all $P>0.05$ ) from baseline in any of the groups after intervention (Table 2). A significant increase in IC was found in both the CET group and the combined training group. However, there was no difference in $\triangle \mathrm{IC}$ between CET group and combined training group after intervention $(P>0.05)$. 
Table 2 Effects of pulmonary rehabilitation in the combined training, CET, and control groups

\begin{tabular}{|c|c|c|c|c|c|}
\hline Parameter & $\begin{array}{l}\text { Control group } \\
(n=26)\end{array}$ & $\begin{array}{l}\text { CET group } \\
(n=27)\end{array}$ & $\begin{array}{l}\text { Combined } \\
\text { training group } \\
(\mathrm{n}=\mathbf{2 8})\end{array}$ & $F$ & $P$-value \\
\hline$\Delta \mathrm{PI}_{\max }\left(\mathrm{cmH}_{2} \mathrm{O}\right)$ & $-2.38 \pm 0.94$ & $1.32 \pm 0.91^{a}$ & $5.20 \pm 0.89^{\mathrm{a}, \mathrm{b}}$ & 17.151 & $<0.001$ \\
\hline$\Delta P E_{\max }\left(\mathrm{cmH}_{2} \mathrm{O}\right)$ & $-5.29 \pm 1.97$ & $5.42 \pm 1.92^{\mathrm{a}}$ & $2.37 \pm 1.88^{\mathrm{a}}$ & 7.943 & 0.001 \\
\hline$\Delta \mathrm{FVC}(\mathrm{L})$ & $0.00 \pm 0.05$ & $-0.02 \pm 0.05$ & $0.11 \pm 0.05$ & 2.211 & 0.117 \\
\hline$\Delta \mathrm{FVC} \%$ pred (\%) & $-2.10 \pm 2.20$ & $-0.69 \pm 2.15$ & $4.83 \pm 2.13$ & 2.832 & 0.065 \\
\hline$\Delta \mathrm{FEV}_{1}(\mathrm{~L})$ & $-0.02 \pm 0.03$ & $0.01 \pm 0.03$ & $0.06 \pm 0.03$ & 0.593 & 0.555 \\
\hline$\Delta$ FEV $\%$ pred (\%) & $1.75 \pm 1.61$ & $0.16 \pm 1.56$ & $1.23 \pm 1.54$ & 0.264 & 0.769 \\
\hline$\Delta \mathrm{FEV} / \mathrm{FVC}$ & $-3.23 \pm 2.00$ & $0.55 \pm 1.96$ & $-0.13 \pm 1.90$ & 1.007 & 0.370 \\
\hline$\Delta \mathrm{MVV}(\mathrm{L})$ & $1.11 \pm 1.20$ & $0.90 \pm 1.18$ & $4.14 \pm 1.17$ & 2.360 & 0.101 \\
\hline$\Delta \mathrm{IC}(\mathrm{L})$ & $-0.04 \pm 0.02$ & $0.06 \pm 0.02^{\mathrm{a}}$ & $0.10 \pm 0.02^{\mathrm{a}}$ & 13.209 & $<0.001$ \\
\hline$\Delta \mathrm{mMRC}$ & $0.11 \pm 0.13$ & $-0.33 \pm 0.13^{a}$ & $-0.47 \pm 0.13^{a}$ & 5.534 & 0.006 \\
\hline$\triangle \mathrm{CAT}$ & $0.30 \pm 0.35$ & $-3.00 \pm 0.34^{a}$ & $-2.39 \pm 0.34^{a}$ & 25.715 & $<0.001$ \\
\hline$\triangle S G R Q$ & $0.95 \pm 0.56$ & $-3.5 I \pm 0.54^{a}$ & $-3.32 \pm 0.54^{a}$ & 20.598 & $<0.001$ \\
\hline$\triangle \mathrm{HADS}$ & $-0.12 \pm 0.39$ & $-3.47 \pm 0.39^{a}$ & $-3.16 \pm 0.38^{\mathrm{a}}$ & 22.410 & $<0.001$ \\
\hline$\Delta$ HADS depression & $-0.15 \pm 0.28$ & $-2.20 \pm 0.27^{a}$ & $-2.10 \pm 0.27^{a}$ & 17.605 & $<0.001$ \\
\hline$\Delta$ HADS anxiety & $-0.10 \pm 0.20$ & $-1.32 \pm 0.20^{\mathrm{a}}$ & $-1.07 \pm 0.20^{\mathrm{a}}$ & 14.470 & $<0.001$ \\
\hline$\Delta \mathrm{BMI}\left(\mathrm{kg} / \mathrm{m}^{2}\right)$ & $-0.02 \pm 0.21$ & $0.54 \pm 0.21$ & $0.23 \pm 0.21$ & 1.712 & 0.187 \\
\hline$\Delta$ FFMI $\left(\mathrm{kg} / \mathrm{m}^{2}\right)$ & $-0.11 \pm 0.24$ & $0.68 \pm 0.24^{\mathrm{a}}$ & $0.87 \pm 0.23^{\mathrm{a}}$ & 4.825 & 0.011 \\
\hline$\triangle \mathrm{BODE}$ & $-0.07 \pm 0.13$ & $-0.02 \pm 0.12$ & $-0.24 \pm 0.12$ & 1.194 & 0.309 \\
\hline
\end{tabular}

Notes: Data are presented as mean \pm SE unless otherwise stated; $\Delta$, difference (after minus before intervention); $P$-value results of analysis of covariance comparison of the differences between groups; ${ }^{a} P<0.05$ vs control group; ${ }^{b}<0.05$ vs $C E T$ group.

Abbreviations: $\mathrm{CET}$, cycle ergometer training; $\mathrm{PI}_{\text {, }}$, maximal inspiratory pressure; $\mathrm{PE}_{\text {ax }}$, maximal expiratory pressure; $\mathrm{FVC}$, forced vital capacity; $\mathrm{FEV}$, forced expiratory volume in I second; \%pred, percent predicted; MVV, maximal voluntary ventilation; IC, inspiratory capacity; mMRC, modified Medical Research Council; CAT, COPD Assessment Test; SGRQ, St George's Respiratory Questionnaire; HADS, Hospital Anxiety and Depression Scale; BMI, body mass index; FFMI, fat-free mass index; BODE, body mass index, airflow obstruction, dyspnea, and exercise capacity index.

\section{Effects of pulmonary rehabilitation on dyspnea, quality of life, nutritional status, and depression and anxiety}

There were no significant differences with regard to BMI or BODE index between groups after training. The CET group and the combined training group demonstrated significant improvements in dyspnea, quality of life, FFMI, and depression and anxiety when compared with the control group $(P<0.05)$. However, there were no significant differences in improvements in these parameters between the CET group and the combined training group after intervention $(P>0.05)$.

\section{Effects of the CET + IMT combination in patients with vs without respiratory muscle weakness}

We performed a subgroup analysis in patients from the combined training group to determine the effect of combined training on patients with or without respiratory muscle weakness. Thus, we allocated the patients in the combined training group to two subgroups based on their respiratory muscle strength. The respiratory muscle weakness was defined as a $\mathrm{PI}_{\max }<60 \mathrm{cmH}_{2} \mathrm{O}$. At baseline, when subgroup analysis was performed in the combined training group, there were no significant differences in $\mathrm{PE}_{\max }$, exercise capacity, static pulmonary function, dyspnea, quality of life, depression and anxiety, or nutritional status between patients with and without respiratory muscle weakness. However, these parameters also demonstrated no significant differences between the two subgroups after training ( $P>0.05$; Table 4$)$. There was no significant differences in $\Delta \mathrm{PI}_{\text {max }}$ between the two subgroups after training $(P>0.05$; Table 4$)$.

\section{Effects of pulmonary rehabilitation in patients with vs without malnutrition}

At baseline, malnutrition was defined as FFMI $\leq 16 \mathrm{~kg} / \mathrm{m}^{2}$ in men and $\leq 15 \mathrm{~kg} / \mathrm{m}^{2}$ in women. A post hoc analysis on effects of pulmonary rehabilitation in patients with versus without malnutrition was performed, pooling subjects from the CET group and combined training group $(n=55,7$ females and 48 males) and reallocating them as subjects with normal nutrition or with malnutrition. There were no significant differences of improvements in respiratory muscle strength, exercise capacity, static pulmonary function, dyspnea, quality of life, depression and anxiety or BMI between two subgroups both before and after training $(P>0.05$; Table 5). 
Table 3 Change in exercise capacity and CPET paraments after pulmonary rehabilitation in the combined training, CET, and control groups

\begin{tabular}{|c|c|c|c|c|c|}
\hline Parameter & $\begin{array}{l}\text { Control group } \\
(n=26)\end{array}$ & $\begin{array}{l}\text { CET group } \\
(n=27)\end{array}$ & $\begin{array}{l}\text { Combined } \\
\text { training group } \\
(\mathrm{n}=28)\end{array}$ & $\boldsymbol{F}$ & $P$-value \\
\hline \multicolumn{6}{|l|}{ 6MWD } \\
\hline$\Delta$ Distance $(\mathrm{m})$ & $-1.64 \pm 4.64$ & $32.55 \pm 4.59^{a}$ & $21.68 \pm 4.5 \mathrm{I}^{\mathrm{a}}$ & 14.320 & $<0.001$ \\
\hline$\Delta$ Borg & $0.35 \pm 0.24$ & $-0.10 \pm 0.24$ & $-0.23 \pm 0.23$ & 1.620 & 0.205 \\
\hline \multicolumn{6}{|l|}{ Rest CPET variables } \\
\hline$\Delta \mathrm{VE}(\mathrm{L} / \mathrm{min})$ & $0.14 \pm 0.23$ & $0.16 \pm 0.23$ & $-0.43 \pm 0.22$ & 2.301 & 0.107 \\
\hline$\Delta \operatorname{Rf}(\mathrm{b} / \mathrm{min})$ & $0.05 \pm 0.31$ & $-0.21 \pm 0.30$ & $-0.69 \pm 0.30$ & 1.666 & 0.196 \\
\hline$\Delta \mathrm{VT}(\mathrm{L})$ & $-0.01 \pm 0.01$ & $0.01 \pm 0.02$ & $-0.01 \pm 0.02$ & 0.630 & 0.535 \\
\hline$\triangle \mathrm{RER}$ & $0.01 \pm 0.01$ & $0.03 \pm 0.01$ & $0.02 \pm 0.01$ & 0.746 & 0.478 \\
\hline$\Delta \mathrm{SPO}_{2}(\%)$ & $0.12 \pm 0.13$ & $0.30 \pm 0.12$ & $0.31 \pm 0.12$ & $0.7 \mid 4$ & 0.493 \\
\hline$\Delta \mathrm{HR}(\mathrm{b} / \mathrm{min})$ & $-1.37 \pm 1.56$ & $-2.64 \pm 1.53$ & $-4.22 \pm 1.49$ & 0.878 & 0.420 \\
\hline \multicolumn{6}{|l|}{ Peak CPET variables } \\
\hline$\Delta \mathrm{VE}(\mathrm{L} / \mathrm{min})$ & $0.91 \pm 0.92$ & $1.96 \pm 0.90$ & $0.20 \pm 0.89$ & 0.989 & 0.377 \\
\hline$\Delta \operatorname{Rf}(\mathrm{b} / \mathrm{min})$ & $0.5 \mathrm{I} \pm 0.6 \mathrm{I}$ & $0.50 \pm 0.60$ & $-0.60 \pm 0.59$ & 1.140 & 0.325 \\
\hline$\Delta \mathrm{VT}(\mathrm{L})$ & $0.01 \pm 0.02$ & $0.00 \pm 0.02$ & $0.01 \pm 0.02$ & 0.057 & 0.945 \\
\hline$\triangle \mathrm{RER}$ & $-0.01 \pm 0.01$ & $0.00 \pm 0.01$ & $-0.02 \pm 0.01$ & 1.089 & 0.342 \\
\hline$\Delta \mathrm{SPO}_{2}(\%)$ & $0.28 \pm 0.44$ & $-0.39 \pm 0.43$ & $0.22 \pm 0.42$ & 0.743 & 0.479 \\
\hline$\Delta \mathrm{HR}(\mathrm{b} / \mathrm{min})$ & $-1.96 \pm 1.83$ & $0.8 \mathrm{I} \pm \mathrm{I} .79$ & $-1.21 \pm 1.76$ & 0.631 & 0.535 \\
\hline$\Delta \mathrm{VO}_{2 \max }(\mathrm{mL} / \mathrm{min})$ & $3.45 \pm 20.96$ & $\mid 49.01 \pm 20.04^{\mathrm{a}}$ & $122.96 \pm 19.47^{a}$ & 14.180 & $<0.001$ \\
\hline$\Delta \mathrm{VO}_{2 \max } \%$ pred (\%) & $0.34 \pm 1.43$ & $9.56 \pm 1.35^{\mathrm{a}}$ & $6.28 \pm 1.32^{\mathrm{a}}$ & 11.143 & $<0.001$ \\
\hline$\Delta \mathrm{VO}_{2 \max } / \mathrm{kg}(\mathrm{mL} / \mathrm{min} / \mathrm{kg})$ & $-0.58 \pm 0.39$ & $2.56 \pm 0.36^{\mathrm{a}}$ & $2.26 \pm 0.36^{a}$ & 21.037 & $<0.001$ \\
\hline$\Delta \mathrm{AT}(\mathrm{mL} / \mathrm{min})$ & $-0.29 \pm 34.72$ & $157.53 \pm 33.19^{a}$ & $104.75 \pm 32.30^{\mathrm{a}}$ & 5.482 & 0.006 \\
\hline$\Delta$ AT\%pred (\%) & $-0.27 \pm 1.96$ & $7.68 \pm 1.84^{\mathrm{a}}$ & $4.90 \pm 1.8 I^{\mathrm{a}}$ & 4.453 & 0.015 \\
\hline$\Delta$ Power (Watt) & $-0.62 \pm 2.29$ & $11.86 \pm 2.25^{\mathrm{a}}$ & $6.10 \pm 2.21^{\mathrm{a}}$ & 7.547 & 0.001 \\
\hline$\Delta$ Borg & $0.08 \pm 0.20$ & $-0.18 \pm 0.20$ & $-0.36 \pm 0.20$ & 1.258 & 0.290 \\
\hline
\end{tabular}

Notes: Data are presented as mean \pm SE unless otherwise stated; $\Delta$, difference (after minus before intervention); $P$-value results of analysis of covariance comparison of the differences between groups; ${ }^{a}<0.05$ vs control group.

Abbreviations: CET, cycle ergometer training; CPET, cardiopulmonary exercise testing; 6MWD, 6-minute walking distance; VE, minute ventilation; Rf, respiratory frequency; VT, tidal volume; RER, respiratory exchange ratio; $\mathrm{SPO}_{2}$, pulse oxygen saturation; $\mathrm{HR}$, heart rate; $\mathrm{VO}_{2 \text { max }}$, maximal oxygen consumption; \%pred, percent predicted; AT, anaerobic threshold.

The $\Delta$ FFMI demonstrated no significant differences between patients with normal nutrition and malnutrition after training $(P>0.05$; Table 5).

\section{Discussion}

The present study demonstrated a significantly greater improvement in inspiratory muscle strength, but not in other measurements including exercise capacity, IC, dyspnea, quality of life, nutritional status, and depression and anxiety, as achieved by CET + IMT compared with CET alone. Furthermore, combined training did not confer an additional benefit in COPD patients with inspiratory muscle weakness when compared with CET alone. While pulmonary rehabilitation could lead to significant improvement in nutritional status of COPD patients, nutritional status did not appear to affect the effects of pulmonary rehabilitation.

Exercise training was shown to improve respiratory muscle strength, exercise capacity, IC, and quality of life, and also relieve dyspnea. ${ }^{29,30}$ Lower limb training represents one of the best available means of exercise training. ${ }^{4}$ However, the training effects in some cases are typically modest, given that a few COPD patients have to stop training or lower their training intensity because of dyspnea during exercise. ${ }^{5,31}$ Therefore, greater pulmonary rehabilitation effects could potentially be achieved should the severity of dyspnea be reduced. IMT can improve the strength and endurance of the respiratory muscles and decrease dyspnea in COPD patients during their training. ${ }^{5}$ However, our study did not indicate that the addition of IMT enhanced the training effect. The reason could be explained by the fact that IMT did not reduce exercise-related dyspnea as indicated by Larson et al, even though it increased strength of the inspiratory muscles. ${ }^{10}$

Wanke et al demonstrated that a combination of CET and IMT could significantly improve inspiratory muscle performance, maximal oxygen uptake, and peak ventilatory pattern when compared with CET alone after 8 weeks of training. ${ }^{11}$ Our findings were inconsistent with those results. 
Table 4 Effects of combined training in patients who had COPD with or without respiratory muscle weakness

\begin{tabular}{|c|c|c|c|c|}
\hline Parameter & $\begin{array}{l}\text { Normal respiratory } \\
\text { muscle group } \\
(\mathrm{n}=19)\end{array}$ & $\begin{array}{l}\text { Weakened respiratory } \\
\text { muscle group } \\
(n=9)\end{array}$ & $\boldsymbol{F}$ & $P$-value \\
\hline$\Delta \mathrm{PI}_{\max }\left(\mathrm{cmH}_{2} \mathrm{O}\right)$ & $6.89 \pm 1.45$ & $1.22 \pm 2.44$ & 2.980 & 0.097 \\
\hline$\Delta P E_{\max }\left(\mathrm{cmH}_{2} \mathrm{O}\right)$ & $0.46 \pm 1.69$ & $4.03 \pm 2.52$ & 1.308 & 0.264 \\
\hline$\triangle 6 \mathrm{MWT}(\mathrm{m})$ & $16.67 \pm 6.70$ & $28.14 \pm 10.70$ & 0.673 & 0.420 \\
\hline$\Delta \mathrm{VO}_{2} \max (\mathrm{mL} / \mathrm{min})$ & $137.93 \pm 24.76$ & $78.60 \pm 37.13$ & 1.642 & 0.212 \\
\hline$\Delta \mathrm{VO}_{2} \max \%$ pred (\%) & $6.65 \pm 1.95$ & $5.63 \pm 2.97$ & 0.073 & 0.790 \\
\hline$\Delta \mathrm{VO}_{2} \max / \mathrm{kg}(\mathrm{mL} / \mathrm{min} / \mathrm{kg})$ & $2.32 \pm 0.34$ & $2.08 \pm 0.50$ & 0.154 & 0.698 \\
\hline$\Delta \mathrm{AT}(\mathrm{mL} / \mathrm{min})$ & $102.50 \pm 38.09$ & $96.72 \pm 57.39$ & 0.006 & 0.937 \\
\hline$\Delta \mathrm{AT} \%$ pred (\%) & $5.38 \pm 2.58$ & $3.87 \pm 3.95$ & 0.091 & 0.766 \\
\hline$\Delta \mathrm{FVC}(\mathrm{L})$ & $0.12 \pm 0.08$ & $0.10 \pm 0.12$ & 0.018 & 0.985 \\
\hline$\Delta$ FVC\%pred (\%) & $6.7 I \pm 3.4 I$ & $2.95 \pm 4.99$ & 0.380 & 0.543 \\
\hline$\Delta \mathrm{FEV}_{1}(\mathrm{~L})$ & $0.02 \pm 0.06$ & $0.15 \pm 0.08$ & 1.789 & 0.193 \\
\hline$\Delta$ FEV $\%$ pred (\%) & $0.01 \pm 2.64$ & $4.66 \pm 3.87$ & 0.966 & 0.335 \\
\hline$\Delta \mathrm{FEV} / \mathrm{FVC}$ & $0.5 I \pm 1.23$ & $0.90 \pm 1.81$ & 0.403 & 0.531 \\
\hline$\Delta \mathrm{MVV}(\mathrm{L})$ & $2.55 \pm 1.94$ & $7.72 \pm 2.83$ & 2.244 & 0.147 \\
\hline$\Delta \mathrm{IC}(\mathrm{L})$ & $0.10 \pm 0.03$ & $0.13 \pm 0.04$ & 0.305 & 0.586 \\
\hline$\Delta \mathrm{mMRC}$ & $-0.53 \pm 0.17$ & $-0.33 \pm 0.25$ & 0.361 & 0.553 \\
\hline$\triangle \mathrm{CAT}$ & $-2.45 \pm 0.32$ & $-2.38 \pm 0.48$ & 0.016 & 0.899 \\
\hline$\triangle S G R Q$ & $-3.19 \pm 0.74$ & $-4.26 \pm 1.15$ & 0.520 & 0.478 \\
\hline$\triangle \mathrm{HADS}$ & $-2.88 \pm 0.40$ & $-4.25 \pm 0.60$ & 3.320 & 0.080 \\
\hline$\Delta \mathrm{HADS}$ depression & $-2.07 \pm 0.35$ & $-3.19 \pm 0.52$ & 3.046 & 0.093 \\
\hline$\triangle \mathrm{HADS}$ anxiety & $-0.94 \pm 0.12$ & $-1.01 \pm 0.18$ & 0.093 & 0.762 \\
\hline$\Delta \mathrm{BMI}\left(\mathrm{kg} / \mathrm{m}^{2}\right)$ & $0.49 \pm 0.30$ & $-0.25 \pm 0.43$ & 1.980 & 0.172 \\
\hline$\Delta$ FFMI $\left(\mathrm{kg} / \mathrm{m}^{2}\right)$ & $1.28 \pm 0.37$ & $0.27 \pm 0.55$ & 2.240 & 0.147 \\
\hline$\triangle \mathrm{BODE}$ & $-0.20 \pm 0.22$ & $-0.47 \pm 0.34$ & 0.410 & 0.528 \\
\hline
\end{tabular}

Notes: Data are presented as mean \pm SE unless otherwise stated; $\Delta$, difference (after minus before intervention); $P$-value results of analysis of covariance comparison of the differences between groups.

Abbreviations: $\mathrm{PI}_{\max }$, maximal inspiratory pressure; $\mathrm{PE}_{\max }$, maximal expiratory pressure; 6MWD, 6-minute walking distance; $\mathrm{VO}_{2 \max }$, maximal oxygen consumption; AT, anaerobic threshold; FVC, forced vital capacity; FEV , forced expiratory volume in I second; \% pred, percent predicted; MVV, maximal voluntary ventilation; IC, inspiratory capacity; mMRC, modified Medical Research Council; CAT, COPD Assessment Test; SGRQ, St George's Respiratory Questionnaire; HADS, Hospital Anxiety and Depression Scale; BMI, body mass index; FFMI, fat-free mass index; BODE, body mass index, airflow obstruction, dyspnea, and exercise capacity index.

Possible reasons for the inconsistent findings across previous studies and ours could be explained by several aspects. First, the respiratory muscle exercise used in the study by Wanke et al included endurance and strength exercises, while strength exercise alone was included in our study. Second, in the study by Wanke et al, respiratory muscle exercise consisted of high-intensity pressure loads during exercise, which was performed four times a week. For strength training, their patients had to maintain pressure of at least $80 \% \mathrm{PI}_{\max }$ in each set. For endurance training, the pressure was $70 \% \mathrm{PI}_{\text {max }}$. Strength training was performed first, and followed by endurance training after at least 10 minutes. In contrast, our study design drew from 2013 ATS/ERS statement on pulmonary rehabilitation, whereby IMT was performed at a load of $30 \% \mathrm{PI}_{\max }$ for each patient at the start of training, which was progressively increased if tolerated. ${ }^{4}$ This exercise intensity had been able to benefit both the respiratory muscle strength and endurance. Therefore, we speculated that whether high-intensity IMT as an adjunct to whole-body exercise training may confer additional benefits in pulmonary rehabilitation needs to be validated by further studies.

Dynamic hyperinflation was common in patients with COPD and changes in IC were used in assessing the level of dynamic hyperinflation. ${ }^{32,33}$ The present study showed that exercise training could increase IC and improve dynamic hyperinflation. However, we found that the addition of IMT did not offer additional effects in terms of IC. The reason could be that the increased respiratory muscle strength still may not effectively improve the abnormal position of diaphragm due to the long-term dynamic hyperinflation.

COPD is known as a chronic systemic inflammatory disease which could lead to severe respiratory symptoms and meanwhile a series of extrapulmonary manifestations, including peripheral skeletal muscle weakness or atrophy, osteoporosis, malnutrition, and depression. As the firstline non-medication treatment, the effects of pulmonary rehabilitation should be evaluated comprehensively. Most 
Table 5 Effects of pulmonary rehabilitation in COPD patients with malnutrition

\begin{tabular}{|c|c|c|c|c|}
\hline Parameter & $\begin{array}{l}\text { Normal nutrition } \\
\text { group } \\
(n=43)\end{array}$ & $\begin{array}{l}\text { Malnutrition } \\
\text { group } \\
(n=12)\end{array}$ & $F$ & $P$-value \\
\hline$\Delta \mathrm{PI}_{\max }\left(\mathrm{mmH}_{2} \mathrm{O}\right)$ & $3.17 \pm 0.90$ & $1.78 \pm 1.73$ & 0.039 & 0.844 \\
\hline$\Delta \mathrm{PE}_{\max }\left(\mathrm{mmH}_{2} \mathrm{O}\right)$ & $4.25 \pm 1.79$ & $-0.95 \pm 3.39$ & $\mathrm{I} .847$ & 0.180 \\
\hline$\Delta 6 \mathrm{MWT}(\mathrm{m})$ & $27.84 \pm 4.21$ & $24.01 \pm 8.02$ & 0.177 & 0.675 \\
\hline$\Delta \mathrm{VO}_{2 \max }(\mathrm{mL} / \mathrm{min})$ & $125.9 \mid \pm 17.34$ & $176.91 \pm 34.03$ & 1.711 & 0.197 \\
\hline$\Delta \mathrm{VO}_{2 \max } \%$ pred (\%) & $7.52 \pm 1.24$ & $9.72 \pm 2.38$ & 0.656 & 0.422 \\
\hline$\Delta \mathrm{VO}_{2 \max } / \mathrm{kg}(\mathrm{mL} / \mathrm{min} / \mathrm{kg})$ & $2.38 \pm 0.28$ & $2.56 \pm 0.53$ & 0.097 & 0.757 \\
\hline$\Delta \mathrm{AT}(\mathrm{mL} / \mathrm{min})$ & $|25.65 \pm 3| .76$ & $|53.5| \pm 65.37$ & 0.134 & 0.716 \\
\hline$\Delta$ AT\%pred (\%) & $6.59 \pm 1.79$ & $5.07 \pm 3.57$ & 0.136 & $0.7 \mid 4$ \\
\hline$\Delta \mathrm{FVC}(\mathrm{L})$ & $0.04 \pm 0.05$ & $0.06 \pm 0.09$ & 0.042 & 0.838 \\
\hline$\Delta \mathrm{FVC} \%$ pred (\%) & $2.73 \pm 1.78$ & $1.21 \pm 3.4$ & 0.156 & 0.694 \\
\hline$\Delta \mathrm{FEV}_{1}(\mathrm{~L})$ & $0.03 \pm 0.03$ & $0.07 \pm 0.06$ & 0.306 & 0.582 \\
\hline$\Delta \mathrm{FEV}, \% p r e d(\%)$ & $0.76 \pm 1.35$ & $1.43 \pm 2.56$ & 0.054 & 0.818 \\
\hline$\Delta \mathrm{FEV} / \mathrm{FVC}$ & $0.86 \pm 0.73$ & $-0.75 \pm 1.39$ & 1.054 & 0.309 \\
\hline$\Delta \mathrm{MVV}(\mathrm{L})$ & $2.51 \pm 1.13$ & $2.76 \pm 2.16$ & 0.01 & 0.919 \\
\hline$\Delta \mathrm{IC}(\mathrm{L})$ & $0.02 \pm 0.02$ & $-0.03 \pm 0.03$ & 1.844 & 0.180 \\
\hline$\Delta \mathrm{mMRC}$ & $-0.43 \pm 0.10$ & $-0.38 \pm 0.19$ & 0.046 & 0.832 \\
\hline$\triangle \mathrm{CAT}$ & $-2.87 \pm 0.25$ & $-2.20 \pm 0.47$ & 1.587 & 0.213 \\
\hline$\triangle \mathrm{SGRQ}$ & $-3.58 \pm 0.4 I$ & $-3.43 \pm 0.78$ & 0.026 & 0.872 \\
\hline$\triangle \mathrm{HADS}$ & $-3.18 \pm 0.27$ & $-3.18 \pm 0.54$ & $<0.001$ & 0.989 \\
\hline$\triangle \mathrm{HADS}$ depression & $-2.03 \pm 0.23$ & $-2.99 \pm 0.47$ & 3.228 & 0.078 \\
\hline$\triangle \mathrm{HADS}$ anxiety & $-1.19 \pm 0.12$ & $-0.92 \pm 0.23$ & 1.015 & 0.318 \\
\hline$\Delta \mathrm{BMI}\left(\mathrm{kg} / \mathrm{m}^{2}\right)$ & $0.37 \pm 0.21$ & $0.36 \pm 0.42$ & 0.001 & $0.98 \mathrm{I}$ \\
\hline$\Delta \mathrm{FFMI}\left(\mathrm{kg} / \mathrm{m}^{2}\right)$ & $0.76 \pm 0.25$ & $0.86 \pm 0.59$ & 0.020 & 0.889 \\
\hline$\triangle \mathrm{BODE}$ & $-0.16 \pm 0.12$ & $-0.10 \pm 0.23$ & 0.049 & 0.825 \\
\hline
\end{tabular}

Notes: Data are presented as mean \pm SE unless otherwise stated; $\Delta$, difference (after minus before intervention); $P$-value results of analysis of covariance comparison of the differences between groups.

Abbreviations: $\mathrm{PI}_{\max }$, maximal inspiratory pressure; $\mathrm{PE}_{\max }$, maximal expiratory pressure; 6MWD, 6-minute walking distance; $\mathrm{VO}_{2 \max }$, maximal oxygen consumption; AT, anaerobic threshold; FVC, forced vital capacity; FEV , forced expiratory volume in I second; \%pred, percent predicted; MVV, maximal voluntary ventilation; IC, inspiratory capacity; mMRC, modified Medical Research Council; CAT, COPD Assessment Test; SGRQ, St George's Respiratory Questionnaire; HADS, Hospital Anxiety and Depression Scale; BMI, body mass index; FFMI, fat-free mass index; BODE, body mass index, airflow obstruction, dyspnea, and exercise capacity index.

of the current studies on pulmonary rehabilitation focus on individual aspects of treatment effects, such as symptoms, exercise performance, or quality of life. In a study by Nikoletou et al, it was highlighted that multiple tests could provide a more comprehensive assessment of muscle functions. ${ }^{34}$ However, those authors evaluated the effects of IMT alone, without comparison to other rehabilitation programs. The sniff inspiratory nasal pressure, a new and main inspiratory muscle test used in that study, was positively correlated with $\mathrm{PI}_{\max }$, and was no better than $\mathrm{PI}_{\max }$ in evaluating respiratory muscle function. In another study, Beaumont et al also attempted to demonstrate the effectiveness of IMT on dyspnea using the Borg scale and multidimensional dyspnea profile questionnaire. ${ }^{35}$ However, in terms of symptom evaluation, they assessed only dyspnea but not other common symptoms in COPD like fatigue, cough, weakness, sleeplessness, and psychological distress. In the present study, several tools (mMRC, CAT, SGRQ, and RPE) were employed for assessment of symptoms. We used $\mathrm{PI}_{\max }$ for assessing respiratory muscle function, incremental cycle ergometer tests for assessing exercise performance, HADS for assessing depression and anxiety, and FFMI and BMI for assessing nutritional status. These efforts were exerted for a comprehensive approach to evaluate the outcomes of pulmonary rehabilitation. We speculated all these measurements, which were rarely used simultaneously in similar studies on this topic, would add to the reliability of our findings.

Reduced inspiratory muscle strength is one of the factors leading to hypoxemia, hypercapnia, dyspnea, and decreased exercise tolerance. ${ }^{36}$ In patients with COPD, inspiratory muscle dysfunction, along with an impaired length-tension relationship, is one of the main contributors to decreased inspiratory muscle strength. ${ }^{12,37-40} \mathrm{~A}$ study reported by Petrovic et al found that IMT improves performance, exercise capacity, and dyspnea, and increases the inspiratory fraction, which is a prognostic factor in patients with COPD. ${ }^{41}$ A meta-analysis that included 32 randomized controlled trials reporting the effects of IMT in patients with COPD 
also found that IMT improves inspiratory muscle strength and endurance, functional exercise capacity, dyspnea, and quality of life. ${ }^{8}$

However, the randomized study by Beaumont et al also concluded that IMT, when used as an adjunct to a 3-week standardized rehabilitation program, was not associated with a significant improvement in either dyspnea or functional parameters in patients with COPD and a $\mathrm{PI}_{\max }>60 \mathrm{cmH}_{2} \mathrm{O} .{ }^{35}$ Although the 2013 ATS/ERS statement on pulmonary rehabilitation indicates that combined exercise may bring additional benefits in COPD patients with respiratory muscle weakness, ${ }^{4}$ the results of the present study showed that IMT may not be useful when combined with CET in this subset of patients. This finding could be explained by the fact that high-intensity lower limb training protocol, used in our study, increased muscle strength and endurance directly and improved respiratory muscle function at the same time. In our subjects, the increased oxygen consumption during exercise caused increased activity of the diaphragm and other accessory respiratory muscles. Furthermore, the intensity of the exercise load met the requirements for pulmonary rehabilitation exercise. ${ }^{33}$ A recent study by Chun et al provides strong evidence to support this view. ${ }^{42}$ They used fluoroscopy-guided chest radiography to assess improvements in diaphragmatic movement before and after pulmonary rehabilitation in 117 patients with COPD, and found significantly improved diaphragmatic movement in both lungs after pulmonary rehabilitation. Therefore, in patients with COPD and respiratory muscle weakness, exercise of the lower limbs improves respiratory muscle function. It is difficult to produce further benefits of exercise in pulmonary rehabilitation by adding IMT.

In this study, BMI and FFMI were used to evaluate the changes of nutritional status before and after pulmonary rehabilitation. Our results showed no significant change in BMI after pulmonary rehabilitation, which was consistent with the previous studies. But we also found a great improvement in FFMI. Our previous studies had shown that FFMI was better than BMI in reflecting the nutritional status of patients with COPD. ${ }^{27}$ When patients undergo high-intensity exercise, the body fat was combusted as major energy. Along with the training, the weight of fat may decrease and muscle mass increase. Although there may not be a significant change in BMI after pulmonary rehabilitation, the proportion of fatfree mass could be increasing. Therefore, we speculated that pulmonary rehabilitation training could improve the nutritional status of COPD patients. In addition, our results also showed that there was no significant difference in exercise tolerance and other indicators between patients with and without malnutrition after pulmonary rehabilitation exercise, suggesting that nutritional status may have little impact on rehabilitation training. However, due to the small number of patients with malnutrition included in this study, this could not be conclusive, thus further studies are needed.

One major limitation of our study was the small number of patients with respiratory muscle weakness. Given that only $20 \%-50 \%$ of patients with COPD have respiratory muscle weakness, as reported in previous studies, ${ }^{5}$ it should not be surprising that there were fewer patients with weakened inspiratory muscle strength than those with normal measurement in the present study. Another limitation is the small number of female patients in training. Further trials should expand the sample of female patients to explore the effect of combined training. Despite these, when compared with previous studies, we included more patients participating in training and used newer indicators to comprehensively evaluate the effects of pulmonary rehabilitation. Even though our results revealed that combined CET and IMT did not confer additional effects, we nevertheless provided evidence about the value of IMT combined with general exercise training. Meanwhile, the present study also offered a basis for the comprehensive assessment of pulmonary rehabilitation by using multiple indicators. Besides, the effects of pulmonary rehabilitation compared in patients with different inspiratory muscle strengths and nutritional status would guide physicians in working-up individualized pulmonary rehabilitation programs.

\section{Conclusion}

The present study showed that combined training is more effective for increasing inspiratory muscle strength when compared with CET alone, but not for improving exercise performance, IC, dyspnea, depressive symptoms, and nutritional status. However, IMT may not be useful when combined with CET in patients with inspiratory muscle weakness. While COPD patients may experience significant improvements in nutritional status after pulmonary rehabilitation, nutritional status did not appear to have any impact on the outcomes of pulmonary rehabilitation. A comprehensive approach can be more objective to evaluate the effects of combined CET and IMT, and may be more meaningful for individualized pulmonary rehabilitation in the future.

\section{Acknowledgments}

The authors are most grateful to Chun-quan Ou PhD (Department of Biostatistics, School of Public Health and 
Tropical Medicine, Southern Medical University, Guangzhou, China) for statistical analysis assistance. This work was supported by Guangzhou Municipal Science and Technology Project (201507020033), Medical Scientific Research Foundation of Guangdong Province (A2016399), Open Project of State Key Laboratory of Respiratory Disease (SKLRD2016OP019), and Clinical Research training program of Southern Medical University (LC2016PY032). The abstract of this paper was presented at the 21 st Congress of the APSR 2016, November 12-15, 2016, Bangkok, Thailand as a poster presentation with interim findings. The poster's abstract was published in "Poster Abstracts" in Respirology.

\section{Disclosure}

The authors report no conflicts of interest in this work.

\section{References}

1. Vogelmeier CF, Criner GJ, Martinez FJ, et al. Global strategy for the diagnosis, management and prevention of chronic obstructive lung disease 2017 report: GOLD executive summary. Respirology. 2017; 22(3):575-601.

2. Barnes PJ, Celli BR. Systemic manifestations and comorbidities of COPD. Eur Respir J. 2009;33(5):1165-1185.

3. Barr RG, Bluemke DA, Ahmed FS, et al. Percent emphysema, airflow obstruction, and impaired left ventricular filling. $N$ Engl J Med. 2010;362(3):217-227.

4. Spruit MA, Singh SJ, Garvey C, et al. An official American Thoracic Society/European Respiratory Society statement: key concepts and advances in pulmonary rehabilitation. Am J Respir Crit Care Med. 2013; 188(8):e13-e64.

5. Hill K, Cecins NM, Eastwood PR, Jenkins SC. Inspiratory muscle training for patients with chronic obstructive pulmonary disease: a practical guide for clinicians. Arch Phys Med Rehabil. 2010;91(9):1466-1470.

6. Bavarsad MB, Shariati A, Eidani E, Latifi M. The effect of home-based inspiratory muscle training on exercise capacity, exertional dyspnea and pulmonary function in COPD patients. Iran J Nurs Midwifery Res. 2015;20(5):613-618.

7. O'Brien K, Geddes EL, Reid WD, Brooks D, Crowe J. Inspiratory muscle training compared with other rehabilitation interventions in chronic obstructive pulmonary disease: a systematic review update. J Cardiopulm Rehabil Prev. 2008;28(2):128-141.

8. Gosselink R, De Vos J, van den Heuvel SP, Segers J, Decramer M, Kwakkel G. Impact of inspiratory muscle training in patients with COPD: What is the evidence? Eur Respir J. 2011;37(2):416-425.

9. Mador MJ, Deniz O, Aggarwal A, Shaffer M, Kufel TJ, Spengler CM. Effect of respiratory muscle endurance training in patients with COPD undergoing pulmonary rehabilitation. Chest. 2005;128(3):1216-1224.

10. Larson JL, Covey MK, Wirtz SE, et al. Cycle ergometer and inspiratory muscle training in chronic obstructive pulmonary disease. Am J Respir Crit Care Med. 1999;160(2):500-507.

11. Wanke T, Formanek D, Lahrmann $\mathrm{H}$, et al. Effects of combined inspiratory muscle and cycle ergometer training on exercise performance in patients with COPD. Eur Respir J. 1994;7(12):2205-2211.

12. Singer J, Yelin EH, Katz PP, et al. Respiratory and skeletal muscle strength in chronic obstructive pulmonary disease: impact on exercise capacity and lower extremity function. $J$ Cardiopulm Rehabil Prev. 2011;31(2):111-119.

13. Pandis N, Chung B, Scherer RW, Elbourne D, Altman DG. CONSORT 2010 statement: extension checklist for reporting within person randomised trials. BMJ. 2017;357:j2835.
14. Schols AM, Ferreira IM, Franssen FM, et al. Nutritional assessment and therapy in COPD: a European Respiratory Society statement. Eur Respir J. 2014;44(6):1504-1520.

15. Gloeckl R, Marinov B, Pitta F. Practical recommendations for exercise training in patients with COPD. Eur Respir Rev. 2013;22(128): $178-186$.

16. Marriott HE, Lamb KL. The use of ratings of perceived exertion for regulating exercise levels in rowing ergometry. Eur J Appl Physiol Occup Physiol. 1996;72(3):267-271.

17. Borg GA. Psychophysical bases of perceived exertion. Med Sci Sports Exerc. 1982;14(5):377-381.

18. American Thoracic Society/European Respiratory Society. ATS/ERS Statement on respiratory muscle testing. Am J Respir Crit Care Med. 2002;166(4):518-624.

19. ATS Committee on Proficiency Standards for Clinical Pulmonary Function Laboratories. ATS statement: guidelines for the six-minute walk test. Am J Respir Crit Care Med. 2002;166(1):111-117.

20. Albouaini K, Egred M, Alahmar A, Wright DJ. Cardiopulmonary exercise testing and its application. Heart. 2007;83(985):675-682.

21. No authors listed. Standardization of Spirometry, 1994 Update. American Thoracic Society. Am J Respir Crit Care Med. 1995;152(3): 1107-1136.

22. Sterk PJ, Fabbri LM, Quanjer PH, et al. Airway responsiveness. Standardized challenge testing with pharmacological, physical and sensitizing stimuli in adults. Report Working Party Standardization of Lung Function Tests, European Community for Steel and Coal. Official Statement of the European Respiratory Society. Eur Respir J Suppl. 1993; 16:53-83.

23. Bestall JC, Paul EA, Garrod R, Garnham R, Jones PW, Wedzicha JA. Usefulness of the Medical Research Council (MRC) dyspnoea scale as a measure of disability in patients with chronic obstructive pulmonary disease. Thorax. 1999;54(7):581-586.

24. Jones PW, Harding G, Berry P, Wiklund I, Chen WH, Kline Leidy N. Development and first validation of the COPD Assessment Test. Eur Respir J. 2009;34(3):648-654.

25. Jones PW, Quirk FH, Baveystock CM, Littlejohns P. A self-complete measure of health status for chronic airflow limitation. The St George's Respiratory Questionnaire. Am Rev Respir Dis. 1992;145(6): 1321-1327.

26. Bjelland I, Dahl AA, Haug TT, Neckelmann D. The validity of the Hospital Anxiety and Depression Scale. An updated literature review. J Psychosom Res. 2002;52(2):69-77.

27. Luo Y, Zhou L, Li Y, et al. Fat-free mass index for evaluating the nutritional status and disease severity in COPD. Respir Care. 2016; 61(5):680-688.

28. Creutzberg EC, Wouters EF, Mostert R, Weling-Scheepers CA, Schols AM. Efficacy of nutritional supplementation therapy in depleted patients with chronic obstructive pulmonary disease. Nutrition. 2003; 19(2):120-127.

29. Yoshimi K, Ueki J, Seyama K, et al. Pulmonary rehabilitation program including respiratory conditioning for chronic obstructive pulmonary disease (COPD): improved hyperinflation and expiratory flow during tidal breathing. $J$ Thorac Dis. 2012;4(3):259-264.

30. Alison JA, McKeough ZJ. Pulmonary rehabilitation for COPD: Are programs with minimal exercise equipment effective? J Thorac Dis. 2014; 6(11):1606-1614.

31. Casaburi R, Patessio A, Ioli F, Zanaboni S, Donner CF, Wasserman K. Reductions in exercise lactic acidosis and ventilation as a result of exercise training in patients with obstructive lung disease. Am Rev Respir Dis. 1991;143(1):9-18.

32. Chen R, Lin L, Tian JW, et al. Predictors of dynamic hyperinflation during the 6-minute walk test in stable chronic obstructive pulmonary disease patients. $J$ Thorac Dis. 2015;7(7):1142-1150.

33. Porszasz J, Emtner M, Goto S, Somfay A, Whipp BJ, Casaburi R. Exercise training decreases ventilatory requirements and exerciseinduced hyperinflation at submaximal intensities in patients with COPD Chest. 2005;128(4):2025-2034. 
34. Nikoletou D, Man WD, Mustfa N, et al. Evaluation of the effectiveness of a home-based inspiratory muscle training programme in patients with chronic obstructive pulmonary disease using multiple inspiratory muscle tests. Disabil Rehabil. 2016;38(3):250-259.

35. Beaumont $\mathrm{M}$, Mialon $\mathrm{P}$, Le Ber-Moy $\mathrm{C}$, et al. Inspiratory muscle training during pulmonary rehabilitation in chronic obstructive pulmonary disease: a randomized trial. Chron Respir Dis. 2015;12(4): 305-312.

36. Charususin N, Gosselink R, Decramer M, et al. Inspiratory muscle training protocol for patients with chronic obstructive pulmonary disease (IMTCO study): a multicentre randomised controlled trial. BMJ Open. 2013;3(8).

37. Levine S, Kaiser L, Leferovich J, Tikunov B. Cellular adaptations in the diaphragm in chronic obstructive pulmonary disease. NEngl J Med. 1997;337(25):1799-1806.
38. Ottenheijm CA, Heunks LM, Li YP, et al. Activation of the ubiquitinproteasome pathway in the diaphragm in chronic obstructive pulmonary disease. Am J Respir Crit Care Med. 2006;174(9):997-1002.

39. Rossi A, Aisanov Z, Avdeev S, et al. Mechanisms, assessment and therapeutic implications of lung hyperinflation in COPD. Respir Med. 2015;109(7):785-802.

40. Hogg JC. Pathophysiology of airflow limitation in chronic obstructive pulmonary disease. Lancet. 2004;364(9435):709-721.

41. Petrovic M, Reiter M, Zipko H, Pohl W, Wanke T. Effects of inspiratory muscle training on dynamic hyperinflation in patients with COPD. Int J Chron Obstruct Pulmon Dis. 2012;7:797-805.

42. Chun EM, Han SJ, Modi HN. Analysis of diaphragmatic movement before and after pulmonary rehabilitation using fluoroscopy imaging in patients with COPD. Int J Chron Obstruct Pulmon Dis. 2015;10: 193-199.

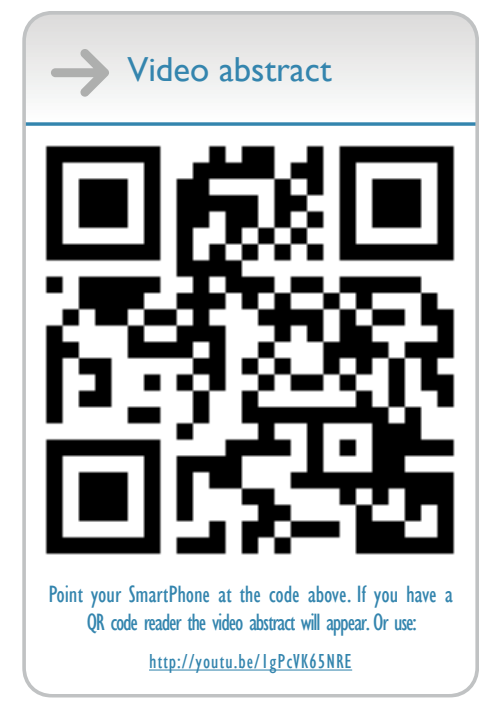

International Journal of COPD

\section{Publish your work in this journal}

The International Journal of COPD is an international, peer-reviewed journal of therapeutics and pharmacology focusing on concise rapid reporting of clinical studies and reviews in COPD. Special focus is given to the pathophysiological processes underlying the disease, intervention programs, patient focused education, and self management protocols.

\section{Dovepress}

This journal is indexed on PubMed Central, MedLine and CAS. The manuscript management system is completely online and includes a very quick and fair peer-review system, which is all easy to use. Visit http://www.dovepress.com/testimonials.php to read real quotes from published authors. 